\title{
LOW ENERGY ELECTRON IRRADIATION INDUCED CHARGING OF DIELECTRIC MATERIALS: MEASUREMENTS AND ANALYSES M. Belhaj, S. Dadouch
}

\author{
ONERA, F-31055 Toulouse, France
}

\begin{abstract}
Charging of dielectric materials under electron irradiation is a commonly encountered problem in many space applications. Spacecraft charging due to solar and cosmic radiations may lead to critical discharge phenomenon. Indeed, under irradiation (especially electron irradiation), insulators as well as floating conductors may charge negatively or positively depending on the incident electron properties (energy, incidence angle, flux ) and on the specific material properties (composition, surface roughness, contamination, temperature, etc.) The knowledge of the electrical properties electron emission yield, conductivity and radiation induced conductivity) under electron, irradiation for each material of the spacecraft is needed for spacecraft plasma interaction software. The energy distribution of the emitted secondary and backscattered electrons was measured dynamically with the help of high-speed hemispherical electron energy analyzer. The evolution of the surface potential of the irradiated sample can be derived from the energy shift of the secondary electron pic. The method is applied to $25-\mu \mathrm{m}$ Kapton.
\end{abstract}

\section{I.INTRODUCTION}

Charging effects due to electron impact is a frequently encountered phenomenon in many space applications $[1,2]$. Spacecraft charging due to solar and cosmic radiations may lead to critical discharge phenomenon. Under irradiation, insulators or floating conductors may charge negatively or positively depending on the incident electron characteristics and the electron emission yield of the material. The study of charging behavior of spacecraft used material is therefore required. Several contactless experimental methods have been developed to measure the trapped charge or the associated surface potential under and after electron irradiation: electron spectrometry [3-5] and X-ray spectroscopy [4], electron mirror method [6], kelvin probe, ... The Kelvin probe and the electron mirror methods can only be used if the charging electron beam is switched off. Therefore, these methods are unable to evaluate the kinetic of charging under irradiation and in some situation (low resistivity dielectric) under estimate the charging magnitude.
The spectroscopic methods have the advantage of being able to measure the surface potential under irradiation. However, Until now, the relatively high acquisition time of the electron energy analyzers (EEA) or X-ray spectrometers impedes the measurement of the evolution of the surface potential during the irradiation. The new generations of EEA equipped with multichannel plate detectors that can operate with a very short acquisition time (second) allow the investigation of charging kinetic. In this contribution the charging kinetic of $25 \mu \mathrm{m}$ Kapton $\mathrm{HN}$ is studied with a help of the electron spectroscopy. The surface potential, Vs, is deduced during the irradiation from the shift of the secondary electron pic.

\section{II.NEGATIVE CHARGING UNDER ELECTRON IRRADIATION}

When an electron beam of energy $E_{0}$, impacts a solid surface, low energy secondary electrons (SE), and backscattered electrons (BSE), with energies ranging from $50 \mathrm{eV}$ to $\mathrm{E}_{0}$, are emitted in the vacuum. If $\mathrm{E}_{0}$ is higher than the second crossover energy $\mathrm{E}_{\mathrm{C} 2}$ or lower than the first crossover energy $E_{C 1}$, a net negative charge is injected in the sample (Electron emission yield, EEY lower than 1). The induced electric field in the vacuum slows the incident electrons and shift their landing energy down to $\mathrm{E}_{0}+\mathrm{eVs},(\mathrm{e}=1.610-19 \mathrm{C})$. This decrease of the impact energy leads to an increase of the electron emission yield. The charging steady state is reached when the $\mathrm{EEY}=1$ and $\mathrm{E}_{0}=\mathrm{E}_{\mathrm{C} 2}$.

\section{EXPERIMENTAL}

\section{A. Experimental setup}

The used experimental setup is shown in Fig. 1. Experiments were made at room temperature and under ultra-high vacuum conditions ( $310^{-10} \mathrm{mbar}$ ). The electron beam of $45^{\circ}$ incidence angle was produced with Kimball Physics ELG2. The used EEA is Aspect from Sigma surface Science. Thanks to multichannel plate detector (128 channels), the EEA is able to acquire (in survey acquisition mode) the electron spectrum in the electron energy range of $0-3.5 \mathrm{keV}$ in few seconds. 


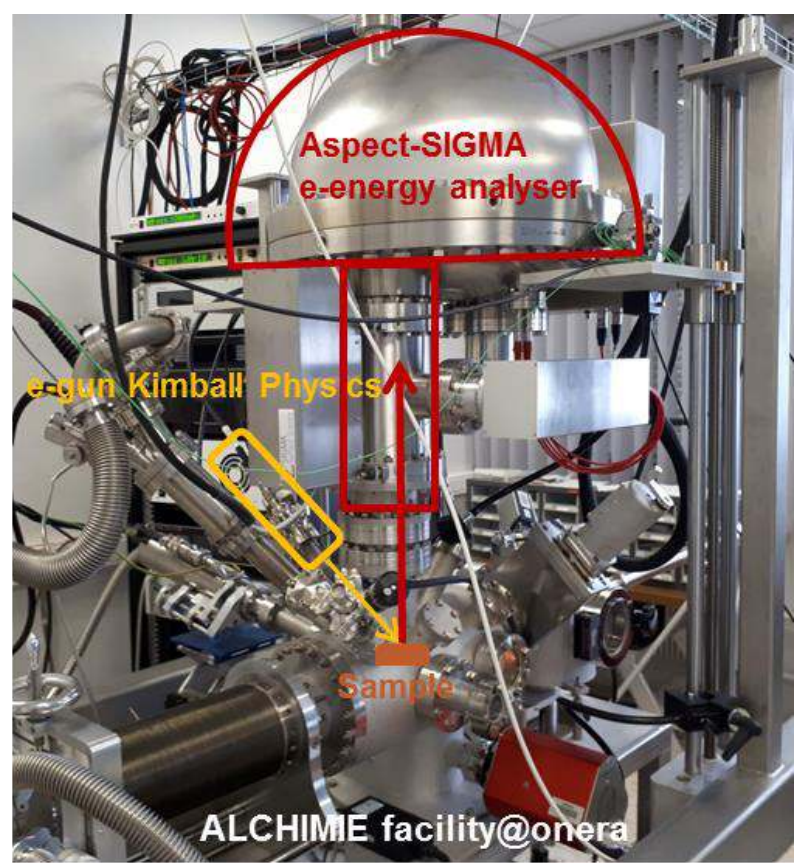

Figure 1. Experimental setup

The Fig. 2 shows an electron energy spectrum measured from the silver plate sample holder at $90 \mathrm{eV}$ incidence energy. The pic at $90 \mathrm{eV}$ is that of the elastically backscattered electrons and the second pic at low energy is associated to the secondary electrons.

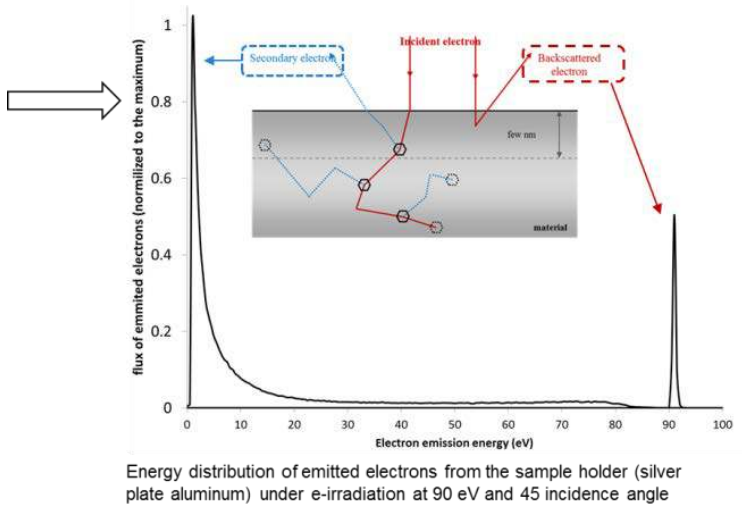

Figure 2. Electron energy distribution of electrons emitted from silver plate sample under electron irradiation at $90 \mathrm{eV}$.

\section{B. Measurement principle}

When the sample is negatively charged, the generated eclectic field between the sample surface and the grounded entrance of the EEY accelerate all the emitted secondary electrons. The onset of the SE pic shifts from about $0 \mathrm{eV}$ to $-\mathrm{eVs}$, here $\mathrm{Vs}$ is the negative surface potential of the sample. To demonstrate the effectiveness of the method, the sample holder was connected to a power supply and in order to induce a controlled a negative surface potential. The SE pics emitted measured under electron beam of $90 \mathrm{eV}$ at $\mathrm{Vs}=0, \mathrm{Vs}=-20 \mathrm{~V}$ and $\mathrm{Vs}=-50 \mathrm{~V}$ are shown in Fig. 3. The SE pics shift to higher energy. Note that the elastic pic remains at $90 \mathrm{eV}$. Indeed, incident electrons that are, first decelerated before the impact with the surface and thereafter accelerated after the backscattering. Therefore, the energy balance is zero.

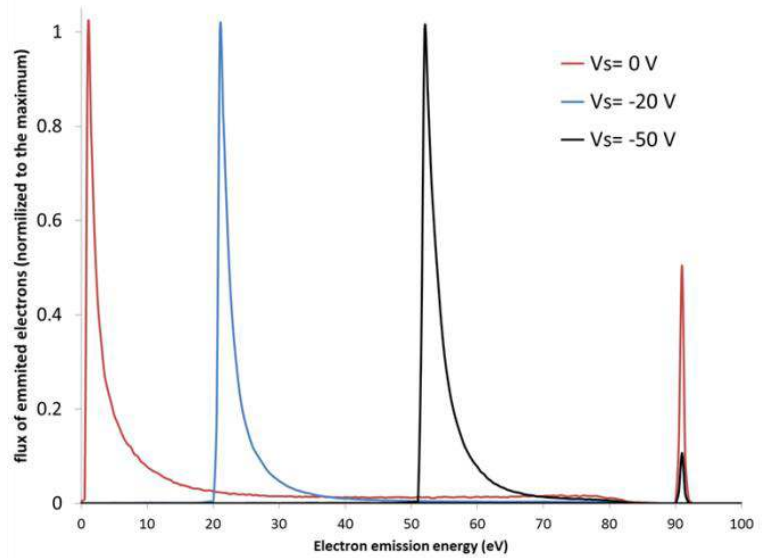

Figure 3. SE pics emitted measured under electron beam of $90 \mathrm{eV}$ at $\mathrm{Vs}=0, \mathrm{Vs}=-20 \mathrm{~V}$ and $\mathrm{Vs}=-50 \mathrm{~V}$

The SE pic onset energy is plotted as function of the applied surface potential on the Fig. 4. The onset energy shift corresponds perfectly to the applied surface potential bias.

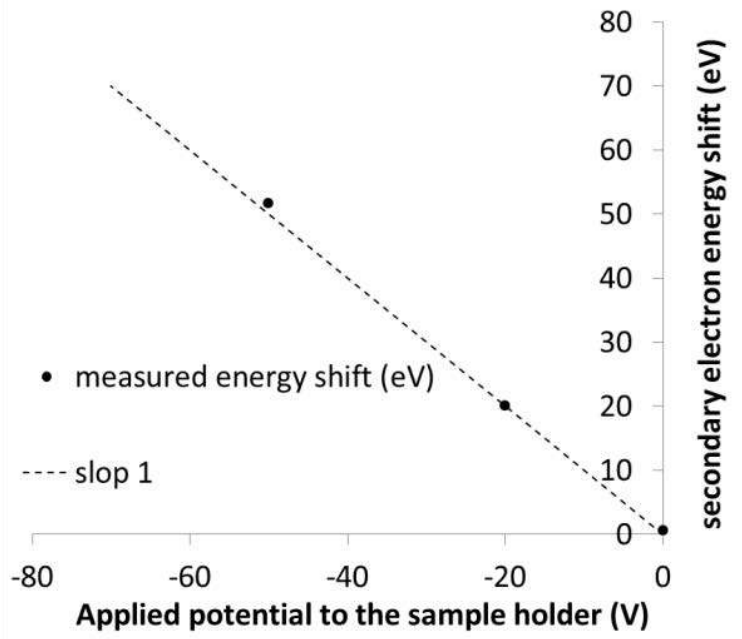

Figure 4. Secondary electron pic energy shift as function of the applied bias to the sample holder

\section{RESULTS}

The Kapton sample (25 $\mu \mathrm{m}$ HN from Dupont) was irradiated with an electron beam of $1.5 \mathrm{keV}$ at $45^{\circ} \mathrm{C}$ and and about $10 \mathrm{nA} / \mathrm{cm}^{2}$. The electron energy distributions were acquired in the fast mode of the EEY (survey mode) every $1 \mathrm{~s}$. The obtained SE pics are shown in the Fig. 5. 


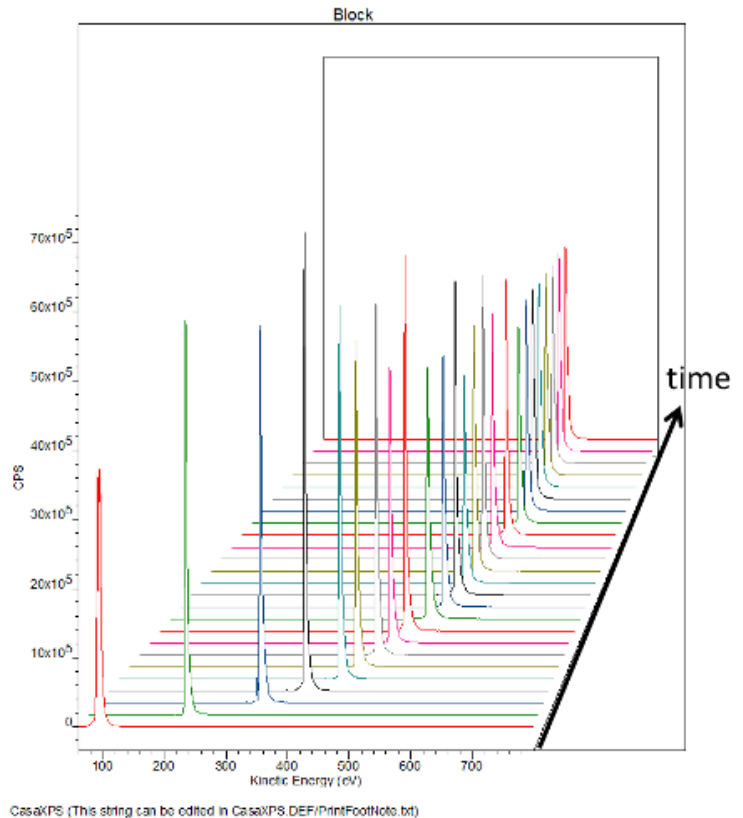

Figure 5. SE pics acquired every 1 sduring the irradiation of kapton at $1.5 \mathrm{keV}$ electron beam.

The evolution of the surface potential as function of the irradiation time deduced from the shift of the SE pic is shown in Fig. 6.

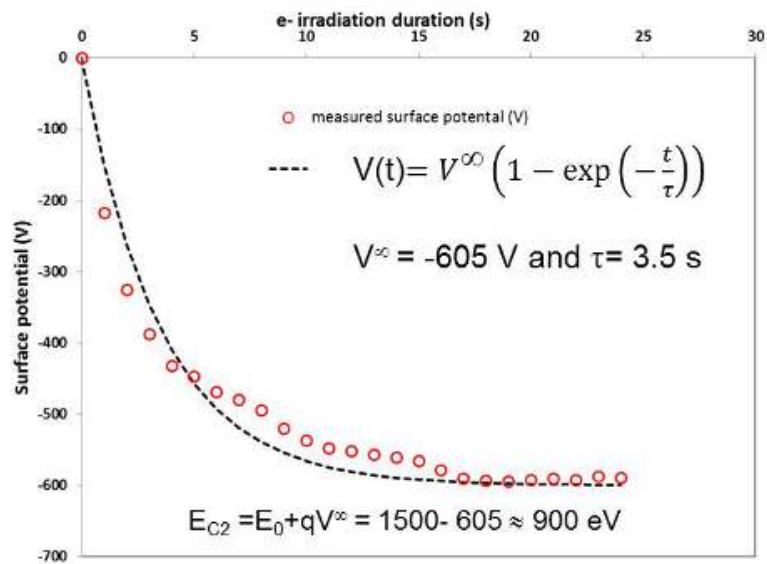

Figure 6. Evolution of the surface potential of as function of the time under $1.5 \mathrm{keV}$ electron irradiation.

The surface potential stars to decrease rapidly at the first steps of irradiation and thereafter reaches a steady state situation with Vs $=-605 \mathrm{~V}$. The impact energy at the steady states about $900 \mathrm{eV}$. If we suppose that the leakage current is negligible, this steady state impact energy should correspond approximately to $\mathrm{E}_{\mathrm{C} 2}$. In previous work [8], we have measured the EEY at normal incidence of Kapton $\mathrm{HN}$ and found $\mathrm{E}_{\mathrm{C} 2}$ about $700 \mathrm{eV}$. Knowing that $\mathrm{E}_{\mathrm{C} 2}$ increases with the incidence angle increases, the present results seems to be in agreement with the EEY properties of Kapton

\section{V.CONCLUSIONS}

The new generation of electron energy analyzer with fast acquisition time capabilities may be very helpful to investigate the negative charging kinetic of dielectrics under electron irradiation. The capabilities of such techniques are illustrated on a kapton sample.

\section{REFERENCES}

[1] J.-C. Mateo-Velez et al., "Spacecraft Worst Case Surface Charging: On the Importance of Measuring the Electron Emission Yield Under Representative Environmental Conditions,"IEEE Trans. Plasma Sci., DOI: 10.1109/TPS.2019.2925435, July 2019.

[2] J.-C. Matéo-Vélez et al., "Spacecraft surface charging induced by severe environments at geosynchronous orbit," Space Weather, vol. 16, no. 1, p.89, Jan. 2018.

[3] A. Melchinger, "Dynamic double layer model: Description of time dependent charging phenomena in insulators under electron beam irradiation,” J. Appl. Phys, vol. 78, p 6224, 1995.

[4] M. Belhaj et al, "Analysis of two methods of measurement of surface potential of insulators in SEM: electron spectroscopy and X-ray spectroscopy methods," Applied surface science, vol. 177, p 58, 2001

[5] O. Jbara et al, "Surface potential measurements of electron-irradiated insulators using backscattered and secondary electron spectra from an electrostatic toroidal spectrometer adapted for scanning electron microscope applications," Review of Scientific Instrument, vol. 22, no. 3, pp. 1788, 2001.

[6] B. Vallayer, "T Space charge measurement in a dielectric material after irradiation with a $30 \mathrm{kV}$ electron beam: Application to single-crystals oxide trapping properties," Review of Scientific Instruments, vol. 70, , p. 3102, 1999.

[7] M. Belhaj et al, "A Kelvin probe based method for measuring the electron emission yield of insulators and insulated conductors subjected to electron irradiation," Journal of Physics D: Applied Physics, vol. 42, no. 10, p. 105309, 2009. 